\title{
Item-specific control of attention in the Stroop task: Contingency learning is not the whole story in the item-specific proportion-congruent effect
}

\author{
Giacomo Spinelli ${ }^{1}$ Stephen J. Lupker ${ }^{1}$ \\ Published online: 8 November 2019 \\ (C) The Psychonomic Society, Inc. 2019, corrected publication 2020
}

\begin{abstract}
A robust finding in the Stroop literature is that congruency effects (i.e., the color-naming latency difference between color words presented in incongruent vs. congruent colors) are larger for color words presented most often in their congruent color than for color words presented most often in incongruent colors. However, the cause of this item-specific proportion congruent (ISPC) effect is unclear, as it might be produced by either a conflict-adaptation strategy (e.g., prepare for conflict when the word RED appears) and/or a more general learning mechanism of stimulus-response contingencies (e.g., prepare to respond blue when the word RED appears). Thus far, attempts to directly dissociate the two processes appear to indicate no role for conflict adaptation, at least in situations in which contingency learning is also possible. We re-examined this conclusion in a Stroop task in which contingency learning and itemspecific conflict frequency were manipulated partially independently. In addition to a contingency-learning effect emerging for stimuli matched on conflict frequency, a conflict-adaptation effect also emerged for stimuli matched on contingency. The two effects also had different time courses, with the contingency-learning effect emerging early and remaining stable throughout the experiment and the conflict-adaptation effect arising later in the experiment. These results challenge not only the contingency-learning account of the ISPC effect, an account that denies the existence of a conflict-adaptation process, but also control accounts that assume that, although conflict-adaptation processes do exist, they are not used when contingency learning is also possible.
\end{abstract}

Keywords Conflict adaptation · Conflict monitoring $\cdot$ Contingency learning $\cdot$ Item-specific proportion-congruent effect $\cdot$ Stroop

\section{Introduction}

An ongoing debate in cognitive control research concerns how the human control system adapts to situations where

The original publication included the following errors which were left uncorrected in the proofing process: 1) in the last paragraph of the Introduction, the sentence "adaptation to conflict may have been the only option at their disposal in those conditions" should have been "adaptation to conflict frequency", etc.; 2) in the second paragraph of the Materials, the examples provided for low-contingency and highcontingency incongruent items in the MI set (i.e., BLUE in green and BLUE in white, respectively), should have been reversed (i.e., BLUE in white and BLUE in green, respectively); 3 ) in Table 1, the word in the last column, "YELLOW", should have been "WHITE"; 4) in Table 3, the first "High contingency MI items" column should have been under RTs, not error rates; 5) in the Results, MSE values were incorrectly reported.

Giacomo Spinelli

gspinel@uwo.ca

Stephen J. Lupker

lupker@uwo.ca

1 Department of Psychology, University of Western Ontario, London, Ontario N6A 5C2, Canada resolution of conflict is frequently required. One such situation was examined by Jacoby, Lindsay, and Hessels (2003), who reported that in the Stroop (1935) task, congruency effects (i.e., the color-naming latency difference between incongruent stimuli, e.g., the word RED presented in yellow, and congruent stimuli, e.g., the word RED presented in red) are larger for words that appear most often in their congruent color (e.g., the word RED presented most often in red) than for words that appear most often in an incongruent color (e.g., the word GREEN presented most often in yellow).

As Jacoby et al. noted, this Item-Specific ProportionCongruent (ISPC) effect might reflect either (or both) of two processes. First, it might reflect use of a conflict-adaptation process in which recognition of specific stimuli regulates the engagement of appropriate control processes - a control explanation (Bugg, Jacoby, \& Toth, 2008). ${ }^{1}$ Specifically, the recognition

\footnotetext{
${ }^{1}$ Note that, in the literature, the term "conflict adaptation" is often used to refer to the congruency sequence effect, a trial-by-trial modulation of the congruency effect (Gratton, Coles, \& Donchin, 1992). However, here, we use this term more generally to refer to any form of conflict-induced adaptive control, including adaptation to the frequency with which conflict occurs for specific items in the task (for a similar use of this term, see Schmidt, 2013b, 2019).
} 
of a frequently conflicting (Mostly Incongruent - MI) word, for example, the word GREEN, would induce focused attention to the color, resulting in reduced interference on incongruent trials. In contrast, the recognition of an infrequently conflicting (Mostly Congruent - MC) word, for example, the word RED, would induce a relaxation of attention. Consequently, word processing would be encouraged, resulting in large interference when the $\mathrm{MC}$ word does conflict with the color.

Alternatively (or additionally), the ISPC effect might reflect the more general process of learning a contingency between a word and the response typically made to that word - a contingency-learning explanation (Schmidt \& Besner, 2008). For example, if the MI word GREEN appears more often in yellow (an incongruent, but high-contingency (i.e., most probable) color for that word) than in green (the congruent, but lowcontingency (i.e., less probable) color for that word), individuals will use the word GREEN to predict a yellow response, thus speeding up responses to GREEN presented in yellow (which will be faster than normal for an incongruent stimulus) but not speeding up responses to GREEN presented in green (which will not be faster than normal for a congruent stimulus). The congruency effect for this word will thus be reduced. Conversely, individuals will use the MC word RED to predict the (congruent) red response. Thus, latencies will speed up when the word and color are congruent, producing a large congruency effect.

In recent years, a number of researchers have tried to dissociate control and contingency-learning accounts of the ISPC effect (Atalay \& Misirlisoy, 2012; Bugg \& Hutchison, 2013; Hazeltine \& Mordkoff, 2014; Hutchison, 2011; Schmidt, 2013a). The conclusion that those researchers have reached is that when a high-contingency color exists for both MC words and MI words (i.e., when not only MC words appear more frequently in their congruent color but also MI words appear more frequently in a specific incongruent color), the ISPC effect that is obtained is entirely produced by contingency learning. That is, item-specific conflict adaptation would not be implemented at all when contingency learning could be reliably used for all the words, as in the type of situation originally examined by Jacoby et al. (2003).

According to Bugg and Hutchison (2013), however, a conflict-adaptation strategy might still be implemented in some situations, for example, when MI words appear in four colors (one congruent and three incongruent) that are equally probable, so that no contingencies exist for those MI words. In order to support this idea, in their Experiment 3, those authors compared a MI condition of this sort to an MC condition in which the $\mathrm{MC}$ words also appeared in four colors (one frequent congruent color and three infrequent incongruent colors). Compared to the $\mathrm{MC}$ words, the $\mathrm{MI}$ words produced a smaller congruency effect (i.e., as expected, there was an ISPC effect just as there is in the standard ISPC paradigm). Crucially, the MI words also produced reduced interference in a new manipulation introduced in the final block of that experiment.
In this final block, in addition to the colors used up to that point in the experiment, a new set of colors was included that had not been used before in the experiment, and both MI and MC words were presented in those new incongruent colors. In this block, latencies for naming, for example, the incongruent color brown (a color used only in the final block of the experiment) were faster if that color appeared in an MI word than if it appeared in an MC word. Bugg and Hutchison (2013) suggested that the shorter latencies for naming these new incongruent colors in MI words in the final block were due to the fact that participants had previously learned to focus attention to the color when those words were presented. This learning process, presumably, occurred as a result of having used a conflict-adaptation strategy in dealing with those MI words earlier in the experiment, when item-specific conflict frequency was being manipulated.

As pointed out by Schmidt (2019), however, the contrast between those MC and MI words is not simply a contrast between words differing in item-specific conflict frequency, but also a contrast between informative words (i.e., the MC words, for which a contingency can be learned between the word and the congruent color) and uninformative words (i.e., the MI words, for which no contingencies can be learned). The reason this second type of contrast is relevant is that informative stimuli are more likely to attract attention than uninformative stimuli (e.g., Jiang \& Chun, 2001). If attention to informative words is increased, those words should produce more interference when presented in incongruent colors (including new incongruent colors, that is, those colors that only appeared in Bugg and Hutchison's (2013) final block). In contrast, if attention to uninformative words is reduced, those words should produce less interference when presented in incongruent colors (including new incongruent colors). The implication is that in Bugg and Hutchison's situation, attention could have been adapted to the informativeness of the words rather than (or in addition to) their item-specific conflict frequency, making the contrast between the type of MI words they used and their more typical MC words not as interpretable as it first appears.

Note that informativeness is not confounded with itemspecific conflict frequency in Jacoby et al.'s (2003) design, as in that design both $\mathrm{MC}$ and MI words are informative (i.e., a high-contingency color exists for both types of words). Because attentional adaptation to word informativeness should not influence performance in Jacoby et al.'s task, their design would thus appear more appropriate to dissociate conflict-adaptation and contingency-learning accounts of the ISPC effect.

Indeed, Schmidt (2013a) does appear to have provided a very straightforward demonstration that item-specific conflict adaptation is not applied in Jacoby et al.'s (2003) ISPC design, a design in which a high-contingency color exists for all of the words being used. To do so, Schmidt constructed a Stroop task in which MC words, for example, RED, and MI words, for example, GREEN, could be compared on what were "contingency-matched" incongruent trials. For example, blue was a 
low-contingency and equally probable color for RED and GREEN. The existence of a conflict-adaptation mechanism would imply that because MC words should induce relaxed attention whereas MI words should induce focused attention to the color, the MC word RED should produce more interference than the MI word GREEN when those words are presented in blue. Performance on MC and MI words when presented in this third color, however, was equivalent, suggesting that no conflict-adaptation strategy was in use. In contrast, a robust contingency-learning effect that cannot be explained in terms of word-specific conflict frequency emerged in the comparison between high-contingency and lowcontingency items, for example, the MI word GREEN in the high-contingency color yellow was responded to faster than the word GREEN in the low-contingency color blue. Overall, these results would appear to offer strong support to the idea that the ISPC effect has "everything to do with contingency" and "nothing to with congruency" (Schmidt \& Besner, 2008).

In the present research, we modified Schmidt's (2013a) dissociation procedure to address a potential problem in his experiment. In his design, the critical comparison for probing conflict adaptation involved a contrast between MC words and MI words when presented in low-contingency incongruent colors. However, this situation was somewhat different than that in the original ISPC paradigm (Jacoby et al., 2003) because Schmidt's contrast was largely based on stimuli in which the word and the color had different proportion congruency statuses. That is, these stimuli involved MI words that were paired with mostly congruent colors (i.e., colors that typically appeared with congruent words) as well as MC words that were often paired with mostly incongruent colors (i.e., colors that typically appeared with incongruent words). For example, GREEN was a word associated with frequent conflict (an MI word); however, in the crucial conditions, it appeared in both blue and red, colors that were associated with infrequent conflict (i.e., MC colors). The reason this issue is relevant is that adaptation to color-specific conflict frequency appears to be possible.

A demonstration of adaptation to color-specific conflict frequency was provided by Bugg and Hutchison (2013) in their Experiment 2, an experiment in which MC colors (e.g., the color red appearing often with the word RED) were compared with MI colors (e.g., the color yellow appearing often with incongruent words) in a situation in which there were little differences in conflict frequency among the words those colors appeared in (i.e., the words would not be considered either MC or MI words). A color-specific PC effect emerged, with a smaller congruency effect for MI colors than for MC colors.

In addition, the MI colors also produced reduced interference in the final block of Bugg and Hutchison's (2013) experiment, a block in which, using a similar manipulation as in their Experiment 3 described above, Bugg and Hutchison included a new set of words and presented both MI and MC colors in those incongruent words. In this block, naming latencies were longer when, for example, the MC color red appeared in the word BROWN (a word used only in the final block of the experiment) than when the MI color yellow appeared in the word BROWN. Notably, because new words were included in this final block, whatever non-conflict learning process related to the word dimension was engaged in the previous part of the experiment (e.g., contingency learning and/or adaptation to stimulus informativeness) could not have produced the pattern of results obtained for the new words in the final block (Schmidt, 2019). The only plausible explanation would thus be adaptation to colorspecific conflict frequency, a process that would induce more focused attention to task-relevant information upon presentation of a frequently conflicting color and relaxed attention upon presentation of an infrequently conflicting color.

As Bugg and Hutchison (2013) obtained an effect of adaptation to color-specific conflict frequency, albeit in a situation in which the words emitted no strong signal for conflict adaptation (because, as noted, there were no large differences in conflict frequency among the words they used in their Experiment 2), it is possible that color-specific conflict frequency would also have a role in situations that allow adaptation to word-specific conflict frequency as well, that is, in typical ISPC paradigms. If that were the case, that is, if adaptation to word-specific and color-specific conflict frequency were concurrently engaged in ISPC paradigms, it would not be clear whether and how participants resolved the contrast between color-specific and word-specific information for the critical items in Schmidt's (2013a) experiment, items for which, as noted, word-specific and color-specific conflict frequency were often inconsistent.

We present the results of an experiment in which this potential problem was removed by constructing a design that was similar to Schmidt's (2013a) with the exception that the stimuli were divided into two non-overlapping sets, an MC set and an MI set. Thus, on the relevant incongruent trials, MC words were presented solely in (other) MC colors and MI words were presented solely in (other) MI colors. With this modification, word-specific and color-specific information, as in the original paradigm (Jacoby et al., 2003), provided convergent signals for either a strategy favoring relaxed attention (with MC stimuli) or a strategy favoring focused attention to the color (with MI stimuli), thus offering a more appropriate situation to test for the existence of a conflict-adaptation mechanism. $^{2}$

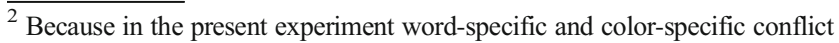
frequency was consistent for all of the stimuli, the term, "item-specific conflict frequency" will be used to indicate the frequency with which conflict occurs on both the word and the color dimensions of the stimuli (i.e., word-specific and/or color-specific conflict frequency). This use of the term is, therefore, not the same as that of most researchers in the field (e.g., Hutchison, 2011; Schmidt, 2013a) who used the term "item-specific conflict frequency" to refer only to word-specific conflict frequency.
} 
This design also constitutes an improvement over other designs aimed at dissociating contingency-learning and conflict-adaptation explanations of the ISPC effect, such as Hutchison's (2011). In the context of a more complex experiment, Hutchison included an ISPC manipulation in which, similar to Schmidt's (2013a) manipulation, MI words appeared in both a high-contingency incongruent color and low-contingency incongruent colors. Consistent with a role of contingency learning in the ISPC effect, MI words produced shorter latencies when presented in the highcontingency incongruent color than in low-contingency incongruent colors. However, the latencies for MI words presented in low-contingency incongruent colors were also somewhat shorter than the latencies for MC words presented in low-contingency incongruent colors. Following Schmidt's (2013a) logic, this result would suggest a role for adaptation to item-specific conflict frequency, in addition to contingency learning, in the ISPC effect.

Unfortunately, the contrast between MC words presented in low-contingency incongruent colors vs. MI words presented in low-contingency incongruent colors in Hutchison's (2011) experiment (a contrast Hutchison did not directly examine) may not be appropriate because, first, the contingency manipulation was somewhat stronger for MC words than for MI words (i.e., the high-contingency color had a $66.67 \%$ probability of occurrence for MC words whereas the highcontingency color had only a $53.33 \%$ probability of occurrence for MI words), unlike the situation in Schmidt's (2013a) experiment in which the manipulation was identical for the two word types (i.e., the high-contingency color had the same probability, $70 \%$, for both MC words and MI words). Thus, although both MC and MI words allowed contingency learning in Hutchison's experiment, the fact that MC words were somewhat more informative (i.e., allowed better learning of contingencies) than MI words means that any contrast between those words might reflect processes related to stimulus informativeness rather than conflict frequency (Schmidt, 2019). Second, in Hutchison's (2011) experiment, similar to what happened in Schmidt's (2013a) experiment, most of the stimuli in the critical contrast (i.e., MC words presented in low-contingency incongruent colors vs. MI words presented in low-contingency incongruent colors) combined MC words with MI colors or, vice versa, MI words with MC colors (e.g., the MI word RED was presented in the low-contingency MC colors yellow and blue). Thus, word-specific and colorspecific conflict frequency were often inconsistent for those crucial stimuli. Neither of these problems, however, affected the present experiment, because, first, MC and MI words were matched in informativeness due to the fact that the contingency manipulation, as in Schmidt's (2013a) experiment, was the same for both words. In addition, as noted, word-specific and color-specific conflict frequency information was consistent for all of the stimuli in the experiment.
Note, finally, that in several paradigms in which a conflictadaptation effect could be potentially produced by contingency-learning biases, conflict-adaptation effects emerged even when those biases were controlled for in the design (e.g., Bugg, 2014; Bugg \& Hutchison, 2013; Crump \& Milliken, 2009; Duthoo, Abrahamse, Braem, Boehler, \& Notebaert, 2014; Spinelli, Perry, \& Lupker, 2019; Weissman, Jiang, \& Egner, 2014). In many of those cases, however, contingency learning was controlled for by negating the opportunity for participants to learn contingencies in at least some of the relevant conditions, with the result being that adaptation to conflict frequency may have been the only option at their disposal in those conditions. In contrast, the situation examined here is one in which contingency leaning can be applied to all of the stimuli in the experiment. Thus, an effect of adaptation to item-specific conflict frequency in the present situation would be one of the first findings of a conflictadaptation effect obtained when a solid alternative strategy contingency learning - could be concurrently applied to the same stimuli, and the first such finding in the ISPC paradigm (although see Shedden, Milliken, Watter, \& Monteiro, 2013, for electrophysiological evidence in support of a conflictadaptation interpretation of the ISPC effect in that paradigm). ${ }^{3}$

\section{Method}

\section{Participants}

Seventy-two students at the University of Western Ontario (age 17-27 years) participated for course credit. This sample size exceeds the sample tested in Schmidt's (2013a) experiment $(N=50)$ and essentially has a power of 1 to detect an effect as large as the conflict-adaptation effect reported by Bugg and Hutchison (2013) in the final block of their Experiment $3\left(\eta_{p}^{2}=.21\right)$, as revealed by a power analysis performed with G*Power 3.1 (Faul, Erdfelder, Buchner, \& Lang, 2009). All participants were native English speakers and had normal or corrected-to-normal vision.

\section{Materials}

Six color names (RED, YELLOW, BLACK, BLUE, GREEN, WHITE) were used as word distractors and the corresponding

\footnotetext{
${ }^{3}$ Although in their Experiment 2, Bugg and Hutchison (2013) did demonstrate an effect of adaptation to color-specific conflict frequency which non-conflict learning processes appear unable to explain, this effect was obtained in a situation in which neither adaptation to word-specific conflict frequency nor contingency learning were valid alternatives. The present experiment improved on Bugg and Hutchison's Experiment 2 because contingency learning, adaptation to word-specific conflict frequency, and adaptation to color-specific conflict frequency could all be concurrently engaged, as in the original ISPC paradigm (Jacoby et al., 2003).
} 
colors (red [R: 255; G: 0; B: 0], yellow [R: 255; G: 255; B: 0], black [R: 0; G: 0; B: 0], blue [R: 0; G: 0; B: 255], green [R: 0; G: 255; B: 0], and white [R: 255; G: 255; B: 255]) were used as target colors. The frequency of word-color combinations in one of the counterbalancings of the experiment is represented in Table 1. The stimuli were divided into two sets, with RED, YELLOW, BLACK, and the corresponding colors forming one set, and BLUE, GREEN, WHITE, and the corresponding colors forming the other set. One set served as the MC set and the other set served as the MI set for each participant. In the MC set, each word appeared in its congruent color 48 times and in each of the two incongruent colors eight times (the item-specific proportion of congruent items was thus $75 \%$ ). Similarly, in the MI set, each word appeared in one incongruent color 48 times and in both the other incongruent color and the congruent color eight times (the item-specific proportion of congruent items was thus $12.5 \%$ ). The contingency manipulations were thus parallel for the words in the MC and MI sets (unlike, e.g., in Hutchison, 2011).

As in Schmidt's (2013a) experiment, three types of incongruent items could be distinguished in this design: (1) lowcontingency incongruent items in the MC set (e.g., RED in yellow in Table 1), (2) low-contingency incongruent items in the MI set (e.g., BLUE in white in Table 1), and (3) highcontingency incongruent items in the MI set (e.g., BLUE in green in Table 1). Note that the first and the second type of incongruent items were matched on contingency (both were low-contingency), with the only difference between them being item-specific conflict frequency. Similarly, the second and third type of incongruent items were matched on item-specific conflict frequency (both were MI items), but differed on contingency. (Item-specific conflict frequency and contingency cannot be dissociated for congruent items, which are all high-contingency in the MC set and all low-contingency in the MI set).

Overall, there were 384 items (168 congruent and 216 incongruent). The assignment of each set to the MC or the MI condition was counterbalanced across participants. The specific incongruent color serving as the high-contingency color for words in the MI set was also counterbalanced across participants.

\section{Procedure}

Each trial began with a fixation symbol ("+") displayed for $250 \mathrm{~ms}$ in the center of the screen followed by a colored word displayed for 2,000 ms or until the participant's response, which was recorded with a microphone connected to the testing computer. Participants were instructed to name the color of the word as quickly and as accurately as possible while ignoring the word itself. Stimuli were presented in uppercase 14point Courier New font, against a medium gray background (R: 128; G: 128; B: 128). No feedback was provided. The experiment was divided into two equal-sized blocks (192 trials per block) with a self-paced pause in the middle. The order of trials within each block was randomized. Initially, participants performed a practice session including six trials in which a string of Xs ("XXXX") was presented in each of the six colors

Table 1 Template for the frequency of color-word combinations

\begin{tabular}{|c|c|c|c|c|c|c|c|}
\hline \multirow[b]{3}{*}{ Color } & & \multicolumn{6}{|l|}{ Word } \\
\hline & & \multicolumn{3}{|c|}{ MC words } & \multicolumn{3}{|c|}{ MI words } \\
\hline & & RED & YELLOW & BLACK & BLUE & GREEN & WHITE \\
\hline \multirow[t]{3}{*}{ MC colors } & Red & 48 & 8 & 8 & & & \\
\hline & Yellow & 8 & 48 & 8 & & & \\
\hline & Black & 8 & 8 & 48 & & & \\
\hline \multirow[t]{3}{*}{ MI colors } & Blue & & & & 8 & 8 & 48 \\
\hline & Green & & & & 48 & 8 & 8 \\
\hline & White & & & & 8 & 48 & 8 \\
\hline
\end{tabular}

Note. The incongruent items shaded in light gray are low-contingency MC items, those shaded in medium gray are low-contingency MI items, those shaded in dark gray are high-contingency MI items 
used in the experiment. The experiment was run using DMDX (Forster \& Forster, 2003) software.

\section{Results}

The waveforms of responses were manually inspected with CheckVocal (Protopapas, 2007) to determine the accuracy of the response and the correct placement of timing marks. Prior to the analyses, invalid trials due to technical failures and responses faster than $300 \mathrm{~ms}$ or slower than the time limit (accounting for .9\% of the data) were discarded. Analyses were performed with SPSS. The default effect-size measure in SPSS, $\eta_{p}^{2}$, is reported as a measure of effect size. Paralleling Schmidt (2013a), a set of three analyses was conducted on both latencies and error rates. First, we conducted a classic ISPC analysis with Congruency (Congruent vs. Incongruent), Item Type (Mostly congruent vs. Mostly incongruent), and Block (First vs. Second) as within-subject factors, with no distinction being made between the low-contingency and the high-contingency incongruent items in the MI set (the data from all incongruent items in the MI set were collapsed). With this analysis, we aimed to demonstrate the presence of an ISPC effect, a pre-condition for conducting the subsequent analyses intended to examine the potential sources of that effect. Second, we conducted a conflict-adaptation analysis (i.e., an analysis testing for the presence of a mechanism of adaptation to item-specific conflict frequency) contrasting low-contingency MC incongruent items and lowcontingency MI incongruent items (i.e., incongruent items matched on contingency) with Item Type (Mostly congruent vs. Mostly incongruent) and Block (First vs. Second) as within-subject factors. Finally, we conducted a contingencylearning analysis (i.e., an analysis testing for the presence of a mechanism of word-response contingency learning) contrasting low-contingency MI incongruent items and highcontingency $\mathrm{MI}$ incongruent items (i.e., incongruent items matched on conflict frequency) with Contingency (Low vs. High) and, again, Block (First vs. Second) as within-subject factors.

Different from Schmidt (2013a), Block was included as a factor in the analyses because there is some evidence in the literature that effects of adaptation to context-specific conflict frequency (of which adaptation to item-specific conflict frequency is a subtype) grow over the course of the experiment (Crump \& Milliken, 2009; see also Jacoby et al., 2003), whereas contingency-learning effects typically arise very early in the experiment and remain stable throughout it (e.g., Lin \& MacLeod, 2018; Schmidt, De Houwer, \& Besner, 2010). Thus, while contingency learning should have a similar effect in the two blocks, an effect of adaptation to item-specific conflict frequency may be more noticeable in the second block than in the first block. The mean response times (RTs) and error rates are presented in Table 2 for the data relevant to the classic ISPC analysis, and in Table 3 for the data relevant to the conflict-adaptation and contingency-learning analyses.

\section{Classic ISPC analysis}

RTs There was a main effect of Congruency, $F(1,71)=$ 273.81, MSE $=5068, p<.001, \eta_{p}^{2}=.794$, indicating faster responses to congruent than incongruent items. There was also an interaction between Congruency and Item type, $F(1,71)=$ $220.59, M S E=1164, p<.001, \eta_{p}^{2}=.757$, reflecting the typical ISPC effect, with a larger congruency effect for MC items $(140 \mathrm{~ms})$ than for MI items $(56 \mathrm{~ms})$. The three-way interaction between Congruency, Item type, and Block was also significant, $F(1,71)=6.90, M S E=869, p=.011, \eta_{p}^{2}=.089$. This interaction indicated that, although there was an ISPC effect (i.e., an interaction between Congruency and Item type) in

Table 2 Mean response times (RTs) and percentage error rates (and corresponding 95\% confidence intervals in parentheses) for the classic ISPC analysis

\begin{tabular}{|c|c|c|c|c|}
\hline \multirow[t]{3}{*}{ Congruency } & \multicolumn{4}{|l|}{ Item type } \\
\hline & \multicolumn{2}{|l|}{ RTs } & \multicolumn{2}{|l|}{ Error rates } \\
\hline & MC items & MI items & MC items & MI items \\
\hline \multicolumn{5}{|l|}{ First block } \\
\hline Congruent & $671[653,689]$ & $703[680,725]$ & $.1[.0, .2]$ & $.0[.0, .0]$ \\
\hline Incongruent & $809[787,831]$ & $769[748,790]$ & $3.0[2.1,3.9]$ & $2.0[1.5,2.5]$ \\
\hline Congruency effect & 138 & 66 & 2.9 & 2.0 \\
\hline \multicolumn{5}{|l|}{ Second block } \\
\hline Congruent & $675[654,695]$ & $718[694,702]$ & $.1[.0, .2]$ & $.5[.0, .9]$ \\
\hline Incongruent & $818[792,843]$ & $764[742,785]$ & $4.6[3.3,5.9]$ & $2.7[2.0,3.4]$ \\
\hline Congruency effect & 143 & 46 & 4.5 & 2.2 \\
\hline
\end{tabular}


Table 3 Mean response times (RTs) and percentage error rates (and corresponding 95\% confidence intervals in parentheses) for the conflict-adaptation and contingency-learning analyses

\begin{tabular}{|c|c|c|c|c|c|c|}
\hline \multirow[t]{3}{*}{ Block } & \multicolumn{6}{|l|}{ Item type } \\
\hline & \multicolumn{3}{|l|}{ RTs } & \multicolumn{3}{|l|}{ Error rates } \\
\hline & $\begin{array}{l}\text { Low-contingency } \\
\text { MC items }\end{array}$ & $\begin{array}{l}\text { Low-contingency } \\
\text { MI items }\end{array}$ & $\begin{array}{l}\text { High-contingency } \\
\text { MI items }\end{array}$ & $\begin{array}{l}\text { Low-contingency } \\
\text { MC items }\end{array}$ & $\begin{array}{l}\text { Low-contingency } \\
\text { MI items }\end{array}$ & $\begin{array}{l}\text { High-contingency } \\
\text { MI items }\end{array}$ \\
\hline First block & $809[787,831]$ & $803[778,829]$ & $763[742,784]$ & $3.0[2.1,3.9]$ & $2.7[1.4,3.9]$ & $1.9[1.4,2.4]$ \\
\hline Conflict adaptation effect & 6 & & & .3 & & \\
\hline Contingency learning effect & & 40 & & & .8 & \\
\hline Second block & $818[792,843]$ & $792[768,817]$ & $759[737,781]$ & $4.6[3.3,5.9]$ & $3.9[2.3,5.6]$ & $2.5[1.9,3.2]$ \\
\hline Conflict adaptation effect & 26 & & & .7 & & \\
\hline Contingency learning effect & & 33 & & & 1.4 & \\
\hline
\end{tabular}

Note. All items in the table are incongruent items. The conflict adaptation effect is the difference between low-contingency MC items and lowcontingency MI items. The contingency learning effect is the difference between low-contingency MI items and high-contingency MI items

both the first block, $F(1,71)=97.08, M S E=949, p<.001, \eta_{p}^{2}$ $=.578$, and the second block, $F(1,71)=157.44, M S E=1084$, $p<.001, \eta_{p}^{2}=.689$, the ISPC effect was smaller in the first block $(\mathrm{MC}$ items $=138 \mathrm{~ms} ; \mathrm{MI}$ items $=66 \mathrm{~ms} ;$ difference $=72$ $\mathrm{ms})$ than in the second block $(\mathrm{MC}$ items $=143 \mathrm{~ms}$; $\mathrm{MI}$ items $=$ $46 \mathrm{~ms}$; difference $=97 \mathrm{~ms}$ ). This difference mainly arose because of the 20-ms reduction in the congruency effect for MI items in the second block compared to the first block, $F(1,71)$ $=6.53, M S E=1171, p=.013, \eta_{p}^{2}=.084$ (the 5 -ms increase in the congruency effect for $\mathrm{MC}$ items in the second block compared to the first block was not significant, $F<1$ ). No other effect reached significance.

Error rates The main effects of Congruency, $F(1,71)=84.05$, $M S E=.001, p<.001, \eta_{p}^{2}=.542$, Item type, $F(1,71)=9.80, M S E$ $=.001, p=.003, \eta_{p}^{2}=.121$, and Block, $F(1,71)=10.15, M S E=$ $.001, p=.002, \eta_{p}^{2}=.125$, were all significant. Those effects indicated more accurate responses to congruent than incongruent items, more accurate responses to MI than $\mathrm{MC}$ items, and more accurate responses in the first block than in the second block, respectively. The interaction between Congruency and Item type was also significant, $F(1,71)=13.55$, MSE $=.001, p<.001, \eta_{p}^{2}=$ .160 , reflecting the typical ISPC effect, with a larger congruency effect for MC items (3.7\%) than for MI items (2.1\%). There was also an interaction between Congruency and Block, $F(1,71)=$ $4.76, M S E=.001, p=.032, \eta_{p}^{2}=.063$, indicating that congruency effects were slightly smaller in the first block $(2.1 \%)$ than in the second block (3.0\%). Finally, the three-way interaction between Congruency, Item type, and Block was marginal, $F(1,71)=3.10$, $M S E=.001, p=.083, \eta_{p}^{2}=.042$. Paralleling the RT data, this marginal interaction indicated a tendency for a smaller ISPC effect in the first block $(\mathrm{MC}$ items $=2.9 \%$; $\mathrm{MI}$ items $=2.0 \%$; difference $=.9 \%)$ than in the second block ( $\mathrm{MC}$ items $=4.5 \%$; MI items $=2.2 \%$; difference $=2.3 \%$ ).

\section{Conflict-adaptation analysis}

RTs The main effect of Block was not significant, $F<1$; however, the main effect of Item type was, $F(1,71)=6.84$, $M S E=2483, p=.011, \eta_{p}^{2}=.088$. Responses to lowcontingency incongruent items in the $\mathrm{MC}$ set $(813 \mathrm{~ms})$ were slower than responses to low-contingency incongruent items in the MI set $(798 \mathrm{~ms})$, indicating an effect of adaptation to item-specific conflict frequency independent from contingency learning. Item type also interacted with Block, $F(1,71)=$ 4.88, MSE $=1417, p=.030, \eta_{p}^{2}=.064$. This interaction indicated that the effect of Item type was smaller in the first block (a nonsignificant 6-ms effect, $t(71)=.84, p=.403, \eta_{p}^{2}=.010$ ) than in the second block (a significant 26-ms effect, $t(71)=$ $3.13, p=.003, \eta_{p}^{2}=.121$ ).

Error rates The only significant effect was that of Block, $F(1$, $71)=5.66, M S E=.003, p=.020, \eta_{p}^{2}=.074$, with more accurate responses in the first block (2.9\%) than in the second block (4.4\%).

\section{Contingency-learning analysis}

RTs The only significant effect was that of Contingency, $F(1$, $71)=47.71, M S E=2047, p<.001, \eta_{p}^{2}=.402$, with faster responses to high-contingency incongruent items in the MI set (761 ms) than to low-contingency incongruent items in the same set $(798 \mathrm{~ms})$. There was no indication that Contingency interacted with Block, $F<1$.

Error rates The main effects of Contingency, $F(1,71)=5.58$, $M S E=.002, p=.021, \eta_{p}^{2}=.073$, and Block, $F(1,71)=4.81$, $M S E=.001, p=.032, \eta_{p}^{2}=.063$, were both significant. Those effects reflected more accurate responses to high-contingency 
incongruent items in the MI set $(2.2 \%)$ than to lowcontingency incongruent items in the same set $(3.3 \%)$, and more accurate responses in the first block $(2.0 \%)$ than in the second block (2.7\%), respectively. Contingency and Block did not interact, $F<1$.

\section{Discussion}

In recent years, several phenomena traditionally interpreted as expressions of a conflict-adaptation mechanism have been reinterpreted invoking more general processes such as feature binding and contingency learning (for reviews, see Schmidt, 2013b, 2019). Jacoby et al. (2003) themselves recognized that the ISPC effect they obtained in the Stroop task (i.e., the finding that MC items elicit larger congruency effects than MI items intermixed in the same list) was compatible with both a conflict-adaptation explanation positing that recognition of specific words regulates the engagement of appropriate control processes and a contingency-learning explanation positing that words are used to anticipate their most likely response. Subsequent work in the ISPC paradigm (Atalay \& Misirlisoy, 2012; Bugg \& Hutchison, 2013; Hazeltine \& Mordkoff, 2014; Hutchison, 2011; Schmidt, 2013a) has led researchers to conclude that contingency learning is the only process involved in the ISPC effect, at least in the situation originally examined by Jacoby et al. in which a high-contingency color exists for both $\mathrm{MC}$ words and MI words.

For the first time, we report evidence challenging that conclusion. Using a design modelled after Schmidt (2013a), we dissociated the individual contributions of adaptation to itemspecific conflict frequency and contingency learning in the ISPC effect by evaluating the impact of conflict adaptation on incongruent items matched on contingency and the impact of contingency learning on incongruent items matched on conflict frequency. We also addressed a problem in Schmidt's (2013a) design (and similar designs such as Hutchison's, 2011), i.e., the fact that words and colors produced divergent, rather than convergent, signals for conflict adaptation in the critical conditions. Specifically, MC words often appeared in MI colors (and vice versa) in Schmidt's (2013a) experiment, whereas in the present experiment, as in Jacoby et al. (2003), all MC words appeared in MC colors and all MI words appeared in MI colors. A regular ISPC effect was obtained when comparing all congruent and incongruent items in the MC and MI sets. More importantly, when the critical types of incongruent items were compared, in addition to a contingency-learning effect emerging when comparing high-contingency and low-contingency incongruent items matched on conflict frequency, a (smaller) conflictadaptation effect also emerged, with slower responses to $\mathrm{MC}$ incongruent items (items that, being typically congruent, should induce relaxed attention and, thus, large interference) than to contingency-matched MI incongruent items (items that, being typically incongruent, should induce focused attention and thus, reduced interference).

The latter result, contrasted with the null effect obtained by Schmidt (2013a), emphasizes the importance in the ISPC paradigm that words and colors provide converging information for attentional control in order for such control to be effectively implemented. More generally, this result challenges not only the contingency-learning account of the ISPC effect, an account which denies the existence of a conflict-adaptation mechanism, but also a recent instantiation of the control account (Bugg \& Hutchison, 2013) which posits that such a mechanism does exist but is not used when contingency learning can be concurrently used, as was the case in our experiment. Thus, even in those situations, both contingency learning and adaptation to item-specific conflict frequency play a role in performance.

Other evidence from our lab is consistent with this conclusion (Spinelli, Krishna, Perry, \& Lupker, in review). In a series of experiments, participants maintained a working-memory load while performing an ISPC paradigm identical to Jacoby et al.'s (2003; i.e., a paradigm in which a high-contingency color existed for both MC words and MI words, but contingency learning and item-specific conflict frequency were not directly dissociable, unlike in the present experiment) and/or a non-conflict version of the Stroop task in which contingencies between color-unrelated words and responses were manipulated so as to parallel those in the ISPC paradigm (e.g., an MI word, for example, BLUE appeared in red three times out of four and in blue one time out of four in the ISPC task and, to parallel that situation, the word SHOP appeared in red three times out four and in blue one time out of four in the nonconflict task).

Maintaining a working-memory load diminished people's ability to learn contingencies in the non-conflict task (i.e., a task in which contingency learning was, presumably, the only process being engaged), consistent with previous findings (also from a non-conflict task), which characterize contingency learning as a process critically dependent on limited-capacity resources (Schmidt et al., 2010). In contrast, maintaining a working-memory load did not alter the ISPC effect in the ISPC task even though parallel contingencies existed in that task. This finding suggests that the ISPC effect reported by Jacoby et al. (2003) does not entirely result from contingency learning, as proposed by many researchers (e.g., Bugg \& Hutchison, 2013; Schmidt \& Besner, 2008), with adaptation to item-specific conflict frequency likely playing an important role as well. The present results, obtained in a design in which contingency learning and item-specific conflict frequency were dissociated directly, complement and extend those conclusions.

Indeed, in addition to obtaining a conflict-adaptation effect independent from contingency learning, we also found that, 
consistent with previous reports of similar effects (Crump \& Milliken, 2009; Jacoby et al., 2003), the conflict-adaptation effect was more pronounced later than early in the experiment (a tendency shared with the overall ISPC effect), suggesting that learning to regulate control in response to item-specific conflict frequency may require some time to develop.

Also consistent with previous reports (e.g., Lin \& MacLeod, 2018; Schmidt et al., 2010), participants acquired the ability to use word-response contingencies quite quickly and, in addition, the use of them remained constant throughout the experiment. The particular situation in which that pattern of results was obtained, however, offers an interesting and somewhat counterintuitive picture. The reason is that the other effect observed here, the conflict-adaptation effect, requires that participants learn to focus attention to the color (and away from the word) in response to MI items (those items used to evaluate the contingency-learning effect), and that effect grew over the course of the experiment. Logically, because contingency learning depends on attention to the predictive dimension (e.g., Jiang \& Chun, 2001), contingency learning should have become less effective as participants' use of a conflictadaptation mechanism grew. Therefore, one might have expected the impact of contingency learning to diminish over the course of the experiment. Nonetheless, there was little indication that the contingency-learning effect was smaller when conflict adaptation was more impactful (later in the experiment) than when conflict adaptation had not yet been developed (early in the experiment).

While further research on the interplay between conflictadaptation and contingency-learning effects is needed, what the present results suggest is that not only does the use of contingency learning not preclude concurrent use of a conflict-adaptation strategy, but reducing attention to words as a result of employing the latter strategy may not limit the effectiveness of concurrent word-response contingency learning. In sum, adaptation to itemspecific conflict frequency and contingency learning may work in a collaborative fashion rather than the adversarial fashion that has often been assumed (e.g., Bugg \& Hutchison, 2013; Schmidt $\&$ Besner, 2008), possibly because they are both expressions of a control system based on associative learning rather than cognitively distinct mechanisms (Abrahamse, Braem, Notebaert, \& Verguts, 2016). For example, contingency learning may offer a rapid way of dealing with conflict (by using the word, even if incongruent with the color, to predict the response) whereas conflict adaptation may only play an important role later, when word-response contingencies have been fully automatized. Future research in the area should allow for a more thorough consideration of these ideas.

Author Note This research was partially supported by Natural Sciences and Engineering Research Council of Canada Grant A6333 to Stephen J. Lupker and the Ontario Trillium Scholarship to Giacomo Spinelli.
Open Practices Statement The raw data and SPSS script used for the analyses are publicly available at https://osf.io/82ukm/. The experiment was not preregistered.

\section{References}

Abrahamse, E., Braem, S., Notebaert, W., \& Verguts, T. (2016). Grounding cognitive control in associative learning. Psychological Bulletin, 142, 693-728. doi:https://doi.org/10.1037/bul0000047

Atalay, N. B., \& Misirlisoy, M. (2012). Can contingency learning alone account for item-specific control? Evidence from within-and between-language ISPC effects. Journal of Experimental Psychology: Learning, Memory, and Cognition, 38, 1578-1590. doi:https://doi.org/10.1037/a0028458

Bugg, J. (2014). Conflict-triggered top-down control: Default mode, last resort, or no such thing? Journal of Experimental Psychology: Learning Memory and Cognition, 40, 567-587. doi:https://doi.org/ $10.1037 / \mathrm{a} 0035032$

Bugg, J. M., \& Hutchison, K. A. (2013). Converging evidence for control of color-word Stroop interference at the item level. Journal of Experimental Psychology: Human Perception and Performance, 39, 433-449. doi:https://doi.org/10.1037/a0029145

Bugg, J. M., Jacoby, L. L., \& Toth, J. P. (2008). Multiple levels of control in the Stroop task. Memory \& Cognition, 36, 1484-1494. doi:https:// doi.org/10.3758/mc.36.8.1484

Crump, M. J., \& Milliken, B. (2009). The flexibility of context-specific control: Evidence for context-driven generalization of item-specific control settings. The Quarterly Journal of Experimental Psychology, 62, 1523-1532. doi:https://doi.org/10.1080/17470210902752096

Duthoo, W., Abrahamse, E. L., Braem, S., Boehler, C. N., \& Notebaert, W. (2014). The congruency sequence effect 3.0: a critical test of conflict adaptation. PloS one, 9, e110462. doi:https://doi.org/10. 1371/journal.pone.0110462

Faul, F., Erdfelder, E., Buchner, A., \& Lang, A. G. (2009). Statistical power analyses using $\mathrm{G}^{*}$ Power 3.1: Tests for correlation and regression analyses. Behavior Research Methods, 41, 1149-1160. doi: https://doi.org/10.3758/BRM.41.4.1149

Forster, K. I., \& Forster, J. C. (2003). DMDX: A Windows display program with millisecond accuracy. Behavior Research Methods, Instruments, \& Computers, 35, 116-124. doi:https://doi.org/10. 3758/BF03195503

Gratton, G., Coles, M. G., \& Donchin, E. (1992). Optimizing the use of information: strategic control of activation of responses. Journal of Experimental Psychology: General, 121, 480-506. doi:https://doi. org/10.1037/0096-3445.121.4.480

Hazeltine, E., \& Mordkoff, J. T. (2014). Resolved but not forgotten: Stroop conflict dredges up the past. Frontiers in Psychology, 5, 1327. doi:https://doi.org/10.3389/fpsyg.2014.01327

Jacoby, L. L., Lindsay, D. S., \& Hessels, S. (2003). Item-specific control of automatic processes: Stroop process dissociations. Psychonomic Bulletin \& Review, 10, 638-644. doi:https://doi.org/10.3758/ BF03196526

Jiang, Y., \& Chun, M. M. (2001). Selective attention modulates implicit learning. The Quarterly Journal of Experimental Psychology A, 54, 1105-1124. doi:https://doi.org/10.1080/02724980042000516

Lin, O. Y. H., \& MacLeod, C. M. (2018). The acquisition of simple associations as observed in color-word contingency learning. Journal of Experimental Psychology: Learning, Memory, and Cognition, 44, 99-106. doi:https://doi.org/10.1037/xlm0000436

Protopapas, A. (2007). Check Vocal: A program to facilitate checking the accuracy and response time of vocal responses from DMDX. 
Behavior Research Methods, 39, 859-862. doi:https://doi.org/10. 3758/BF03192979

Schmidt, J. R. (2013a). The Parallel Episodic Processing (PEP) model: Dissociating contingency and conflict adaptation in the item-specific proportion congruent paradigm. Acta Psychologica, 142, 119-126. doi:https://doi.org/10.1016/j.actpsy.2012.11.004

Schmidt, J. R. (2013b). Questioning conflict adaptation: proportion congruent and Gratton effects reconsidered. Psychonomic Bulletin \& Review, 20, 615-630. doi:https://doi.org/10.3758/s13423-0120373-0

Schmidt, J. R. (2019). Evidence against conflict monitoring and adaptation: An updated review. Psychonomic Bulletin \& Review, 1-19. Advance online publication.

Schmidt, J. R., \& Besner, D. (2008). The Stroop effect: why proportion congruent has nothing to do with congruency and everything to do with contingency. Journal of Experimental Psychology: Learning, Memory, and Cognition, 34, 514-523. doi:https://doi.org/10.1037/ 0278-7393.34.3.514

Schmidt, J. R., De Houwer, J., \& Besner, D. (2010). Contingency learning and unlearning in the blink of an eye: A resource dependent process. Consciousness and Cognition, 19, 235-250. doi:https:// doi.org/10.1016/j.concog.2009.12.016
Shedden, J. M., Milliken, B., Watter, S., \& Monteiro, S. (2013). Eventrelated potentials as brain correlates of item specific proportion congruent effects. Consciousness and Cognition, 22, 1442-1455. doi: https://doi.org/10.1016/j.concog.2013.10.002

Spinelli, G., Krishna, K., Perry, J. R., Lupker, S. J. (in review). Working memory load dissociates contingency learning and item-specific proportion-congruent effects.

Spinelli, G., Perry, J. R., Lupker, S. J. (2019). Adaptation to conflict frequency without contingency and temporal learning: Evidence from the picture-word interference task. Journal of Experimental Psychology: Human Perception and Performance, 45, 995-1014. https://doi.org/10.1037/xhp0000656

Stroop, J. (1935). Studies of interference in serial verbal reactions. Journal of Experimental Psychology, 18, 643-662. doi:https://doi. org/10.1037/h0054651

Weissman, D. H., Jiang, J., \& Egner, T. (2014). Determinants of congruency sequence effects without learning and memory confounds. Journal of Experimental Psychology: Human Perception and Performance, 40, 2022-2037. doi:https://doi.org/10.1037/a0037454

Publisher's note Springer Nature remains neutral with regard to jurisdictional claims in published maps and institutional affiliations. 